\title{
Are there kinetic advantages of GLUT2 in pancreatic glucose sensing?
}

\section{R. Sweet, F. M. M atschinsky}

D epartment of B iochemistry and B iophysics, and D iabetes R esearch Center, U niversity of Pennsylvania, Philadelphia, Pennsylvania, USA

The classical view of pancreatic beta-cell glucose sensing involves a high capacity facilitated glucose transporter, which nearly equalizes the intra- and extracellular glucose signal and glucokinase, which phosphorylates glucose at a rate that is very sensitive to the concentration of glucose in the physiological range. This view was first substantiated by the studies of M atschinsky and E llerman in 1968 [1] and has since been widely accepted as fitting the findings in rat and mouse islets which contain large quantities of GLUT2. However, a recent report by De Vos et al. [2], states that human islets contain little GLUT2, and express G LUT 1 at levels allowing rates of transport over phosphorylation about $10 \%$ of those found in mouse and rat islets. This forced us to address the issue of whether the properties of GLUT2 are inherently suited to the proper function of glucose sensing in the rat, and whether GLUT1 acts analogously to G L U T 2 or would impose the distinct glucose sensing characteristics of human pancreatic beta cells. We did this by quantitative analysis of the kinetic characteristics of G LUT 1 and G LUT 2 using a mathematical model that focuses on the interaction between transport and phosphorylation. Thus, we do not address the metabolon theory of tunneling between transport and glucokinase put forth by Newgard et al. $[3,4]$, the regulation of the transporter protein levels by transcription, translation and degradation, or the possible regulation of glucokinase levels by G LU T2.

Corresponding author: Dr. F.M.M atschinsky, U niversity of Pennsylvania, D iabetes R esearch Center, 501 Stemmler $\mathrm{H}$ all, 36th and $\mathrm{H}$ amilton Walk, Philadelphia, PA 19104-6015, U SA
B eta-cell model of transporter and phosphorylation

Since the extent to which transport affects the rate of glycolysis is mediated through its influence on the intracellular concentration of glucose, a simple model of transport and phosphorylation is adequate for the analysis (Fig.1). Phosphofructokinase, and subsequent glycolytic reactions can only affect the rate of phosphorylation via feedback on hexokinase which appears to be present at only $10 \%$ of glucokinase levels [5] and was therefore ignored. The model used for transportacross the membranewasa simplefacilitated carrier model and for phosphorylation it was a description of glucokinase represented as a $\mathrm{H}$ ill equation.

The premise of carrier protein mediated transport is that the binding site exists in two states, each accessible to one side of the membrane (Fig.1). Transformation between the two states must be separated by a finite energy barrier which differentiates a carrier from a pore. The driving force is the concentration gradient existing across the membranes, and interconversion between the two states when a molecule is bound to the transporter results in movement of the molecule across the membrane. This model does not describe all transporters but, like the M ichaelisMenten model for enzymes, is the simplest and a good starting point.

If the binding of glucose and the change between the conformations is fast compared to the changes in glucose in solution, then the transporter species will reach steady state. Equations 1 and 2 describe the unidirectional (plasma to beta cell) and net rate of glucose transport in terms of 4 parameters, 3 resistances $(R)$ and one affinity parameter $(K)$, instead of the 8 rate constants in Figure 1. A lthough there are $4 \mathrm{Rs}$, only 3 are independent since $R_{00}+R_{e-}$ $\mathrm{e}=\mathrm{R}_{12}+\mathrm{R}_{21}$. The kinetic definitions of $\mathrm{K}$ and the $\mathrm{R} \mathrm{S}$ are given in Stein [6] and have the units of $\mathrm{mmol} / \mathrm{l}$ and $\mathrm{g} \cdot \mathrm{min} / \mu \mathrm{mol}$, respectively. 


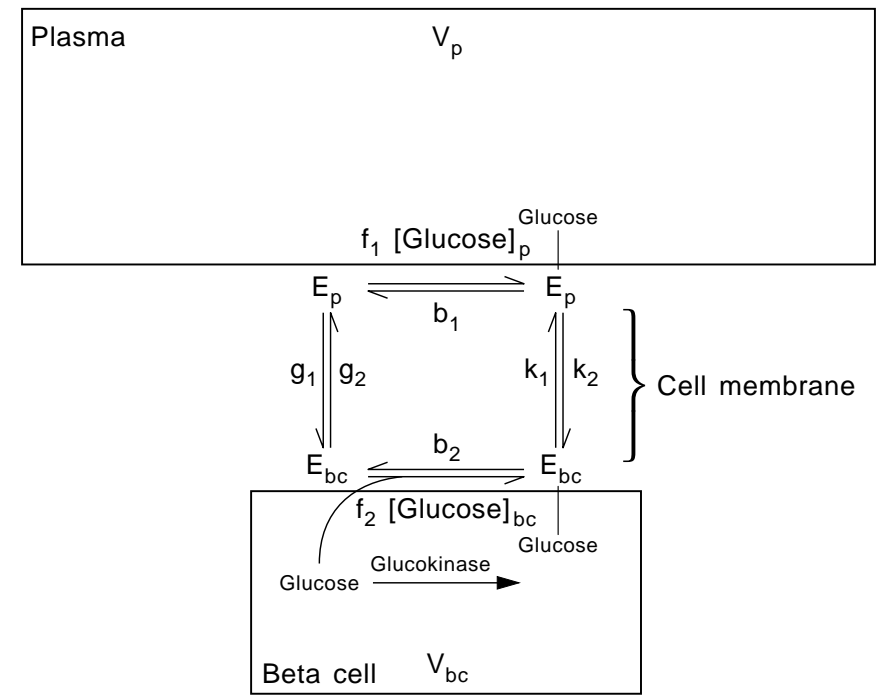

Fig. 1. Model of intracellular glucose in the beta cell, which is governed by kinetic properties of transport and phosphorylation. Transport is assumed to be facilitated by a carrier protein that exists in two conformations, one with the binding site accessible to the extacellular and the other to the intracellular solution. In the formulation of the transport flux equation, it is assumed that all the rate constants $f, g, b$, and $k$ are fast enough relative to the changes in glucose in solutions so that a steady state in the transporter species occurs [5]. The rate of phosphorylation is regulated by glucokinase and since there is no feedback on this enzyme this model completely determines the rate of phosphorylation as a function of the extracellular glucose concentration

$\mathrm{V}^{\mathrm{p}->\mathrm{bc}}=$

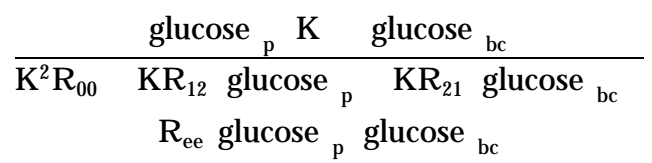

$$
\begin{aligned}
& \int^{p->b c}=
\end{aligned}
$$

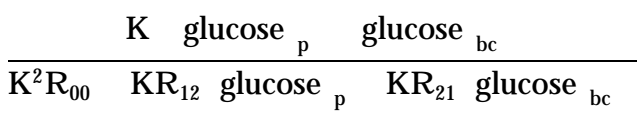

$$
\begin{aligned}
& R_{\text {ee }} \text { glucose }{ }_{p} \text { glucose } b c
\end{aligned}
$$

These equations as they stand are difficult to characterize, so it is useful to make measurements under certain experimental conditions that result in simplified forms. The most common of these are equilibrium exchange and zero trans. A $n$ equilibrium exchange experiment is a measurement of the rate of transport of labelled substrate made when the concentrations of unlabelled substrate on both sides of the membrane are equal. The zero trans is done by measuring an initial rate of transport while there is no substrate on the opposite side of the membrane. Both are measurements of unidirectional flux so that results can be parameterized by a $\mathrm{V}_{\max }$ and $\mathrm{a}$ $\mathrm{K}_{\mathrm{m}}$. Shown in Table 1 are the $\mathrm{V}_{\text {max }}$ and the $\mathrm{K}_{\mathrm{m}}$ in
Table 1. Simplification of transport model (equation 1 ) for certain conditions in terms of $\mathrm{V}_{\text {max }}$ and $\mathrm{K}_{\mathrm{m}}$

\begin{tabular}{lll}
\hline Experiment type & $\mathrm{V}_{\max }$ & $\mathrm{K}_{\mathrm{m}}$ \\
\hline $\begin{array}{lll}\text { Zero trans } \\
\text { plasma to cytosol }\end{array}$ & $1 / \mathrm{R}_{12}$ & $\mathrm{~K} * \mathrm{R}_{00} / \mathrm{R}_{12}$ \\
$\quad$ cytosol to plasma & $1 / \mathrm{R}_{21}$ & $\mathrm{~K} * \mathrm{R}_{00} / \mathrm{R}_{21}$ \\
E quilibrium exchange & $1 / \mathrm{R}_{\text {ee }}$ & $\mathrm{K} * \mathrm{R}_{00} / \mathrm{R}_{\text {ee }}$ \\
\hline
\end{tabular}

terms of the R $\mathrm{s}$ and $\mathrm{K}$ of equation (1) for the three types of experiment. It is important to note that in general a transporter cannot be characterized by only a $\mathrm{V}_{\max }$ and $a \mathrm{~K}_{\mathrm{m}}$, except for these special cases (or as seen below when all the resistances are equal). Thus, to refer to the $\mathrm{K}_{\mathrm{m}}$ of a transporter, doesn't make sense without referring to the conditions of the experiment.

Since the rate of phosphorylation by glucokinase is related to the concentration of glucose in a sigmoidal fashion, a Hill-type equation was used:

$$
V^{G K}=\frac{V_{\max }^{G K} \text { glucose }_{b c}{ }^{h n}}{S_{05}^{G K}{ }^{h n} \text { glucose }_{b c}{ }^{h n}}
$$

where $\mathrm{hn}$ is the $\mathrm{H}$ ill number. The parameters were kept constant throughout the study and were $\mathrm{V}_{\text {max }}=0.7 \mu \mathrm{mol} \cdot \mathrm{min}^{-1} \cdot \mathrm{g}^{-1}, \quad \mathrm{~S}_{0.5}=10 \mathrm{mmol} / \mathrm{l}$, and $\mathrm{hn}=1.7$, except where stated otherwise.

\section{Contrast between G L UT 1 and G L UT2}

In liver and pancreatic beta cells in rats and mice, where GLUT2 is thought to be the predominant transporter, the concentrations of glucose on each side of the membrane are nearly the same $[1,7]$. In both tissues, glucokinase is the predominant phosphorylating enzyme. GLUT1, which is found in the brain and erythrocytes, is associated with a high affinity hexokinase. The major differences between the kinetic characteristics of G LUT 1 and G LUT 2 are that G LU T 2 has a lower affinity and G LUT 1 is asymmetric. Important questions are, is there a kinetic advantage in the association between G L UT 2 and glucokinase, and whether GLUT1 can function in concert with glucokinase as a glucose sensing mechanism in the pancreatic beta cell.

The parameters in equation (1) for G LUT1 have been measured extensively. From experiments on erythrocytes summarized by Carruthers [8], $R_{12}=8 R_{\text {ee, }}$ and $R_{21}=2 R_{\text {ee }}$ and $K=1.5$. The resistances are listed as a factor times $R$ ee since the capacity of an erythrocyte will not be the same for the beta cell. GLUT2 has not been studied as intensively as G LU T1. Studies from both the liver [9] and beta cell 
[10] indicate that the affinity parameter, $K$, is at least 10 times higher than that for GLUT1, and that it is symmetric $\left(R_{12}=R_{21}\right)$. H owever, the ratio of $R_{12}$ to $R_{\text {ee }}$ is not well characterized. It is known to be bigger than 1, though, since trans acceleration was observed in the dog liver [11] and this only occurs when $R_{12}$ is greater than $R_{\text {ee. }}$ In this study it was assumed that $R_{12}=R_{21}=2 R_{\text {ee, }}$ and $K=17$. U sing the zero trans data from $D e$ Vos et al. [2] in human beta cells, $R_{12}=0.4 \mathrm{~g} \cdot \mathrm{min} / \mu \mathrm{mol}\left(\mathrm{V}_{\max }\right.$ for zero trans was five times bigger than the $V_{\max }$ for glucokinase).

B ecause of the complicated nature of these transporters, a simple interpretation of experimental data is not possible. A s discussed above, GLUT1 is asymmetric and displays trans acceleration. A zero trans experiment with GLUT1 yields a $\mathrm{K}_{m}$ and a $\mathrm{V}_{\max }$ that is smaller and larger respectively by a factor of 8 than that of an equilibrium exchange $\left(R_{12}=8 R_{\text {ee }}\right)$. If the physiologic conditions more closely reflect an equilibrium exchange (i.e. if phosphorylation is slow relative to transport) then making a conclusion directly based on a zero trans experiment will be spurious. Thus, the rates of phosphorylation and transport are inextricably linked and their integration via mathematical modelling is necessary to interpret experimental results correctly and quantitatively.

To gain insight from the modelling analysis, it is helpful to keep the following concepts in mind. A t steady state, the net transport flux equals the rate of phosphorylation, and the rate of both will depend on the intracellular glucose concentration, since this determines the rate through glucokinase. The concentration of intracellular glucose is determined by the balance between the rate of depletion (governed by glucokinase and transport out of the cell) and the rate of repletion (governed by transport into the cell). For a finite rate of phosphorylation, there is always less glucose in the cell than outside. The rate of phosphorylation is dependent on all the parameters determining the rate of transport and phosphorylation. Thus, when interpreting the extent to which transport limits phosphorylation, the ratios of $\mathrm{V}_{\text {max }}$ must be considered in conjunction with the ratios of affinities and the glucose concentration.

\section{Optimal value of $\mathrm{K}$}

To examine the effect of the affinity parameter, we will make the common assumption that all the resistances are equal. (A Ithough this is not the case for either G LUT 1 or G LUT2, despite its widespread use.) Equations (1) and (2) simplify to

$$
v^{p->b c}=\frac{\frac{1}{R} \text { glucose }_{p}}{K \quad \text { glucose }_{p}}
$$
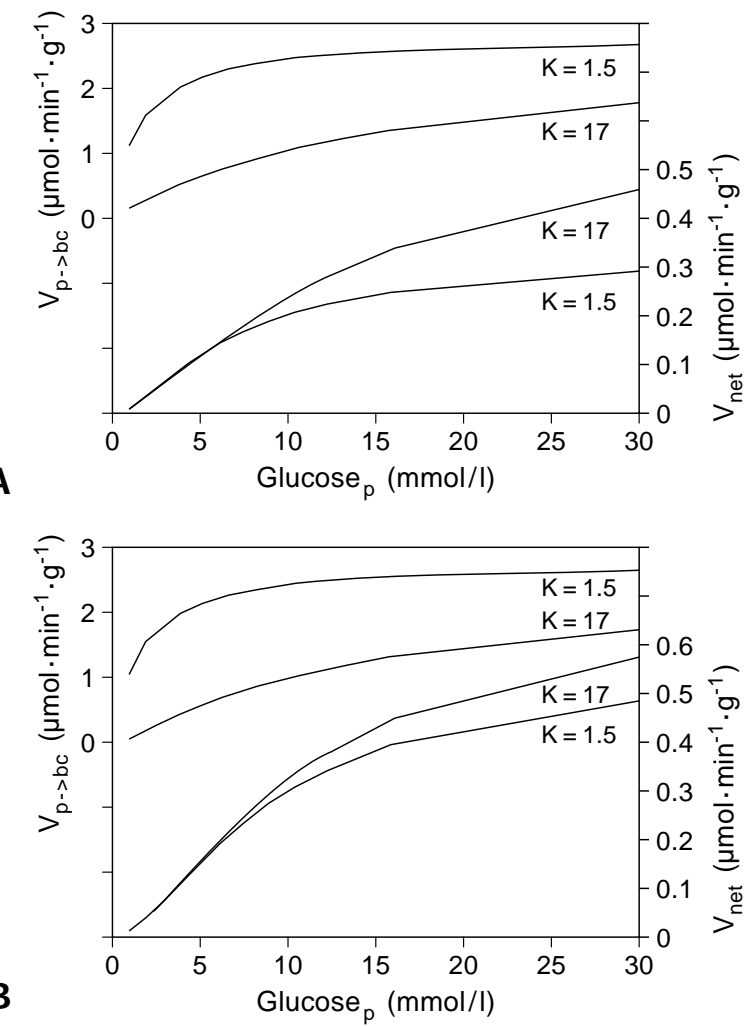

Fig. 2.A,B Glucose dependency curve of unidirectional $\left(V_{p \rightarrow b c}\right)$ and net $\left(V_{n e t}\right)$ steady-state flux of glucose for high and low affinity. Note that at steady state, the net rate of transport is equal to the rate of phosphorylation. All resistances for transport are assumed to be equal ( $R_{12}=R_{12}=R_{\text {eei }}$ see text). The unidirectional flux is higher for the higher affinity, but the net flux is decreased. Ratio of transport maximal activity to that of phosphorylation is $\mathbf{A}: 4 ; \mathbf{B}: 20$. The effect is less pronounced at the higher ratio

and

$J^{p->b c}=\frac{\frac{1}{R} \text { glucose }_{p}}{K \quad \text { glucose }_{p}}-\frac{\frac{1}{R} \text { glucose }_{b c}}{K \quad \text { glucose }_{b c}}$

where $V_{\max }=1 / R$ and $K_{m}=K$. N ote, there is only one $V_{\text {max }}$ and $K_{m}$ for a transporter, irrespective of the conditions. This is because the rate of unidirectional flux is governed by a M ichaelis M enten type dependency on the concentration of the substrate, with no influence of the concentration on the trans side. The difference between this model and a M ichaelis M enten reaction is its bidirectionality, which has consequences that are not immediately apparent. The equations (3-5) were solved as previously described $[12,13]$ and Figure 2 shows the steady state of unidirectional and net flux of glucose into the beta cell at $\mathrm{K}=1.5$ and $17 \mathrm{mmol} / \mathrm{l}$. Note that in the glucose range of 6 to $40 \mathrm{mmol} / \mathrm{l}$, the unidirectional rate of transport is higher for the higher affinity, but the net flux is lower. H owever, below $6 \mathrm{mmol} / \mathrm{l}$, both the unidirectional 


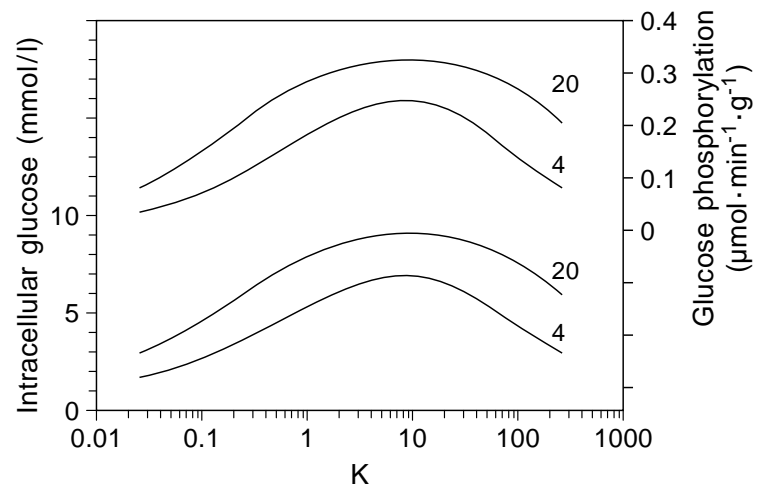

Fig. 3. Effect of affinity on intracellular glucose (bottom curves) and the rate of glucose phosphorylation (or net rate of transport, top curves) at steady state when extracellular glucose is equal to $10 \mathrm{mmol} / \mathrm{l}$. A II transport resistances are assumed to be equal $\left(R_{12}=R_{12}=R_{\text {ee; }}\right.$ see text). N umbers next to curves refer to the ratio of maximal transport activity to phosphorylation. Glucokinase: $V_{\text {max }}=0.7 \mu \mathrm{mol} \cdot \mathrm{min}^{-1} \cdot \mathrm{g}^{-1}$; $\mathrm{S}_{0.5}=10.0 ; \mathrm{H}$ ill number $=1.7$

and the net flux is higher for the high affinity transporter. The effect of increasing the ratio of transport to phosphorylation is to increase the unidirectional rates of transport while decreasing the sensitivity to the affinity (Fig. 2B). Thus, unlike the unidirectional $M$ ichaelis $M$ enten kinetics where an increase in affinity necessarily results in an increase in flux, increasing the affinity of a transporter in this system can result in a lowering of the phosphorylation rate. This is because the rate of exit via the transporter is in direct competition with the phosphorylation of glucose. The higher the ratio of transporter affinity relative to the affinity of glucokinase, the higher the fraction of intracellular glucose that will be exported rather than phosphorylated. So the optimal value of $K$ represents a balance between the enhanced rate of uptake that a high affinity favours and the increased reflux out of the cell. Figure 3 shows the steady-state concentration of glucose and glucose net flux plotted as a function of $K$, for extracellular glucose $=10 \mathrm{mmol} / \mathrm{l}$. A t low $\mathrm{K}$, the flux increases as the $\mathrm{K}$ increases, peaks when $\mathrm{K}$ is between $8-12 \mathrm{mmol} / \mathrm{l}$ and then decreases. The explanation for this behaviour is as follows. When the affinity is low relative to the concentration of extracellular glucose, then transport into the cell limits the rate of phosphorylation. When the affinity for transport is much lower than that of glucokinase, and at concentrations of glucose far above the $K$, the transporter is saturated in both directions so that the fluxes will both be near to the $V_{\text {max }}$ allowing only reduced amounts of phosphorylation to occur. Intracellular glucose would have to reach very low levels to reduce transport out of the cell, which glucokinase is unable to do because of its higher affinity. When the affinity of transport and glucokinase and the glucose concentration in solution is near the $K$, then the rate of unidirectional flux is very sensitive to changes in glucose. The glucose concentration gradient across the membrane can be large enough to allow net flux in transport, while the intracellular glucose levels will be high enough to produce high rates of phosphorylation.

H owever, although there is an optimal range, the curve between 1 and 20 is relatively flat and there is only about a $20 \%$ decrease from the optimal at $\mathrm{K}=1.5$. Thus, the optimal range of $\mathrm{K}$ for glucose transport is about 8-12 $\mathrm{mmol} / \mathrm{l}$, but the loss of activity is not very large a little outside this range.

$\mathrm{U}$ nlike M ichaelis M enten kinetics, where the higher the affinity the higher the activity, in a bidirectional transport/phosphorylation system, a higher affinity can result in a lower activity. L achaal et al. [14] recently achieved similar numerical results using the model in Figure 1 and equations (4-5) for transport and concluded that the high $\mathrm{K}_{m}$ of G LUT2 does not explain its role in insulin secretion. 0 ur results are in agreement since the effect of affinity is small, but the lower affinity of GLUT2 is kinetically better suited to the task of equalizing glucose on opposite sides of the membrane.

\section{E ffect of asymmetry and trans acceleration}

A salient feature of the G LU T1 transporter is that its $V_{\max }$ for zero trans entry is four times slower than that of exit. Thus, for a system that always has less glucose inside the cell than outside due to phosphorylation, one would expect more of this type of transporter would be needed to result in a given level of equalization. This was confirmed by simulations (data not shown), where at low ratios of transport to phosphorylation activity, an asymmetric transporter with identical properties of G LUT 1 except that the asymmetry is reversed, resulted in intracellular levels of glucose and rates of glucose phosphorylation that were higher than that with G LUT1. These differences steadily diminished as the rate of transport activity to that of phosphorylation was increased. Thus, the asymmetry of GLUT1 reduces its efficiency in functioning as an equilibrative transporter.

B oth G L UT 1 and G L U T 2 display trans acceleration, which is the increase in unidirectional flux due to an increase in substrate concentration on the trans side of the membrane. Trans acceleration is measured as an increase in the rate of flux of tracer concentrations of radiolabelled glucose in the presence of cold glucose in non-tracer amounts on the trans side. This will occur when either $R_{12}$ or $R_{21}$ is greater than $R_{\text {ee }}$. $N$ ote that the unidirectional flux is not a physiologic parameter, and only the net flux is important to cell function. However, in order to see whether there is any underlying change in the kinetics of net flux when this phenomenon occurs, intracellular glucose and glucose phosphorylation were simulated as a 


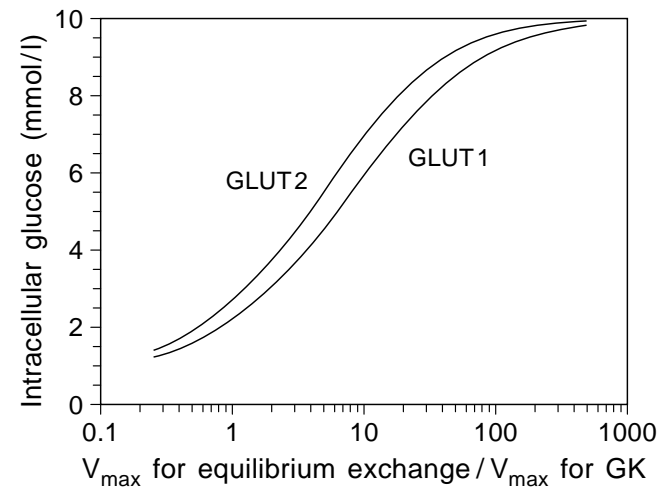

Fig. 4. E ffect of ratio of transport maximal activity (at equilibrium exchange) to that of phosphorylation on the steady-state intracellular concentration of glucose when the extracellular concentration is $10 \mathrm{mmol} / /$ for GLUT1 and GLUT2. For GLUT1: $R_{12}=2 R_{12}=8 R_{\text {ee }} \cdot K=1.5$. For GLUT2: $R_{12}=R_{12}=$ $2 R_{\text {ee. }} \mathrm{K}=17$. G lucokinase: $\mathrm{V}_{\max }=0.7 \mu \mathrm{mol} \cdot \mathrm{min}^{-1} \cdot \mathrm{g}^{-1} ; \mathrm{S}_{0.5}=$ 10.0; $\mathrm{H}$ ill $\mathrm{N}$ umber $=1.7$

function of $K$ using equations 1 and 2 for transport with $R_{12}=R_{21}=2 R_{\text {ee }}$ (data not shown). This is representative of the kinetics of GLUT2, and the results were similar to that in Figure 3. Therefore, unlike the asymmetry of GLUT1, trans acceleration does not lead to a transporter that is less suited to the function of an equilibrating transporter. It seems G LU T 2 has an affinity that is very near the optimal for equilibrating the glucose concentrations across the membrane in the physiologic range.

\section{O verriding influence of capacity}

Both asymmetry and a high affinity are disadvantageous for the task of equilibrating glucose in the presence of intracellular phosphorylation. However, at the values thought to correspond to G L U T1, these effects are not too great and can be overcome by simply raising the ratio of transport protein to phosphorylation enzyme. Figure 4 shows the effect of capacity on the simulated steady-state levels of intracellular glucose for G L U T 1 and G L U T2, calculated using equations (1-3) when the extracellular glucose is $10 \mathrm{mmol} / \mathrm{l}$. For both G LUT 1 and G L U T2, intracellular glucose tends to $10 \mathrm{mmol} / \mathrm{l}$ at high rates of transport. Thus, this demonstrates the important point that if there is enough GLUT1 it will perfom the same function as GLUT2. It can be seen that in the presence of GLUT2, there is always a higher level of intracellular glucose (and hence a higher rate of phosphorylation) than in the presence of GLUT1. However, these two cases are not directly comparable, since for an asymmetric transporter with trans acceleration, there is no global measure of capacity that can be used as a basis for comparison. The $V_{\text {max }}$ for equilibrium exchange was used here, but we could have also used the $V_{\text {max }}$ for zero trans. GLUT 1 does have an impaired ability to equilibrate glucose across the membrane (due to its asymmetry and high affinity), but from the curves in Figure 4, it would not be correct to directly estimate a percent reduction.

The curves can be used to estimate the amount of G L UT 1 or G LU T 2 needed to reduce its rate-limiting effects. It is important to understand that when glucokinase is not saturated, in theoretical terms, transport is necessarily a rate-limiting step. So the question is not whether transport is limiting, it is to what extent it is limiting. H owever, practically, it can be said that if the capacity is high enough, say, to produce intracellular glucose levels greater than $9.0 \mathrm{mmol} / \mathrm{l}(90 \%$ of its equilibrium value) transport would not impose its kinetic characteristics on the glucose sensing system. This occurs at a ratio of $\mathrm{V}_{\max }$ for equilibrium exchange to $\mathrm{V}_{\text {max }}$ of phosphorylation of 80 for G LUT1 and 50 for G LUT2. In the rat, beta cells are thought to have a ratio of 60-100 [15], so the glucose sensing system behaviour is shaped, in practical terms, wholly by glucokinase. A t these levels of transporter, either G LUT 1 or G LUT2 would function in a similar fashion. $D$ e Vos et al. [2] found that the ratio in human islets was 5, when using the $V_{\text {max }}$ for zero trans. Since $R_{12}=8 R_{\text {ee, }}$ this corresponds to a ratio of 40 for our curves which use the $V_{\text {max }}$ for equilibrium exchange as a ratio of transport capacity to glucokinase. This is a factor of 2 lower than that needed for $90 \%$ equilibrium to be reached. H owever, it is likely that isolation and handling of the islets, and the measurement error itself could contribute to an error of a factor of 2 . Thus, although the asymmetry and the low affinity of the GLUT 1 transporter is slightly less suited to the task of equalization of glucose across the membrane, it is not clear that the presence of GLUT1 would result in a different underlying glucose sensing mechanism than that found in the rat, where glucokinase and glucose usage dose response curves are nearly superimposable. If this is not the case, then it is the low capacity of GLUT1 that would be the factor that most distinguishes it from GLUT2. I ts inherent differences would then depend on the factors controlling its gene expression.

G lucose vs insulin secretion with G L UT 1 and G L UT2

To understand how a system with a low amount of transporter (GLUT1 or G LUT2) would behave, the effect of capacity on the glucose sensing-insulin secretion was examined using the model of Sweet and $M$ atschinsky [12]. This model has a function which relates the rate of phosphorylation of glucose to the rate of insulin secretion; it is not truly mechanistic, since there are too many unknowns, however, it does describe the glucose-insulin secretion dose-response characteristics. N ote that there may be postphosphorylation factors that influence the rate of 


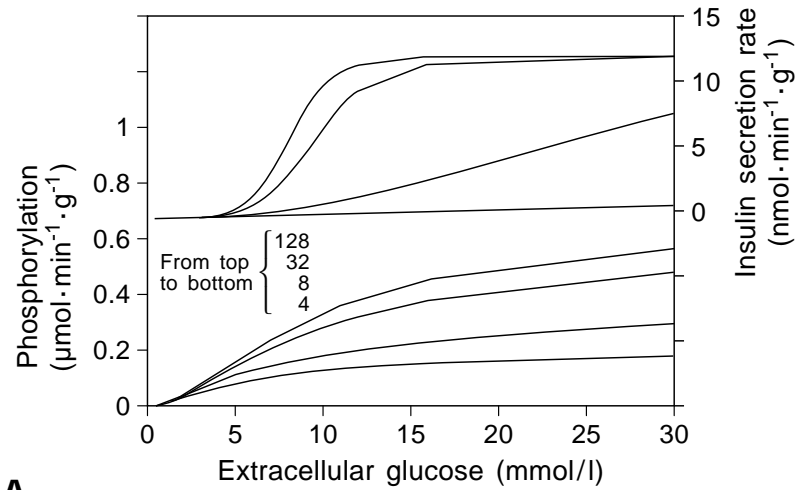

A

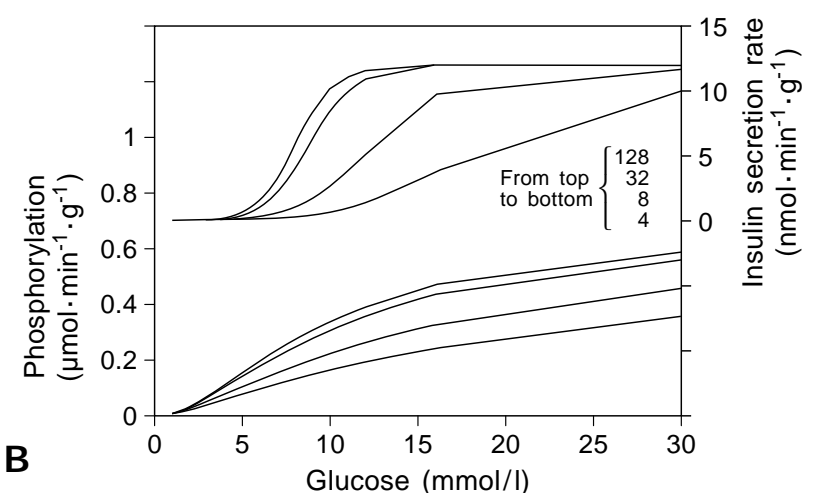

Fig. 5. A,B Effect of ratio of transport maximal activity (at equlibrium exchange) to that of phosporylation on the steadystate rate of phosphorylation and insulin secretion for GLUT1 (A) and GLUT2 (B). R atio of GLUT1 in human islets as measured by $D$ e Vos et al. [2] is about 40 , and for rats and mice the ratio for GLUT 2 is $60-100$

insulin secretion, but this relation is considered constant. The capacity of transport was varied and the steady-state rate of phosphorylation and insulin secretion was plotted as a function of glucose for different values for the $V_{\text {max }}$ for equilibrium exchange $\left(=1 / R_{\text {ee }}\right)$. These plots, shown in Figure $5 \mathrm{~A}$ and $\mathrm{B}$ for GLUT1 and GLUT2 respectively, must be interpreted carefully since they are a mixture of rigorous analysis and speculation. The bottom curve shows the rate of phosphorylation, which at high rates of transport is simply the unadulterated glucokinaseglucose dependency curve. This occurs when the intra- and the extracellular concentrations are nearly equal. A s the capacity decreases, the rate of phosphorylation decreases, as one would expect. It is surprising, though, that there is very little change in the glucose concentration at which the sensitivity is highest (i.e. the concentration of extracellular glucose leading to half-maximal phosphorylation (or the inflection point) does not change appreciably). This is important, because the crucial design of the glucose sensing by beta cells is the dramatic dependency of phosphorylation on glucose in its physiologic range. So even if GLUT1 had a low capacity, from a purely kinetic perspective, transport and phosphorylation could work in concert as a glucose sensor that converts the extracellular glucose signal into an intracellular one in the form of the rate of phosphorylation. On the other hand, the absolute sensitivity of phosphorylation to transport capacity decreases, so that how much transport is needed is governed by the sensitivity of the metabolic coupling factors.

There is a large gain in the conversion of the phosphorylation signal to the secretion of insulin. A change from 5 to $7 \mathrm{mmol} / \mathrm{l}$ glucose $(40 \%)$ can lead to a 10-fold increase in insulin secretion. By relating the rate of insulin secretion with the phosphate potential (A TP/A D P) using a $\mathrm{H}$ ill equation with a $\mathrm{H}$ ill number of 30 , the simulated rate of insulin secretion resembles a threshold effect that triggers at a phosphorylation rate of about $0.15 \mu \mathrm{mol} \cdot \mathrm{min}^{-1} \cdot \mathrm{g}^{-1}$ and has a very high gain. This equation, though empirical, fits insulin secretion data from isolated perifused islets [13] and can illustrate some conceptual points. The top curves of Figure $5 A$ and $B$ were generated from the model and show the rates of insulin secretion that correspond to phosphorylation at the different rates of transport. A t a constant gain for the metabolic coupling factor, a lowering of the transport rate leads to a dramatic decrease in the rate of insulin secretion. A t transport rates over 32, the shape of the glucose insulin secretion curve has the characteristics of a normal response. So in the absence of compensatory changes in the post-metabolic events, a transport capacity below that would lead to drastic impairment in insulin release. However, since the phosphorylation curves retain their sensitivity between 5 and $10 \mathrm{mmol} / \mathrm{l}$ glucose, an increase in the gain of the post-metabolic response could normalize the insulin secretion response. The point here is that the limits at which transport is too low for proper glucose sensing may be governed by the sensitivity of post-metabolic coupling and the ability to compensate by upregulating the gain.

\section{D ominant role of glucokinase in glucose sensing}

We have previously demonstrated the high control strength of glucokinase in the control of the rate of glycolysis based on the known kinetic characteristics of transport, glucokinase and glycolysis [12, 13]. A s we have pointed out, there may be interactions between GLUT2 and other factors, most notably glucokinase, that govern beta-cell metabolism in ways that were not considered. H owever, if our hypothesis of a high capacity transporter (relative to glucokinase) is correct, then the sensitivity of glucose metabolism to transport will be low. It is this feature that results in the relatively small effect of transport affinity and asymmetry on the rate of phosphorylation. To reiterate, the phosphorylation rate was simulated at two different $\mathrm{S}_{0.5} \mathrm{~s}$ for glucokinase, for both G LUT 1 


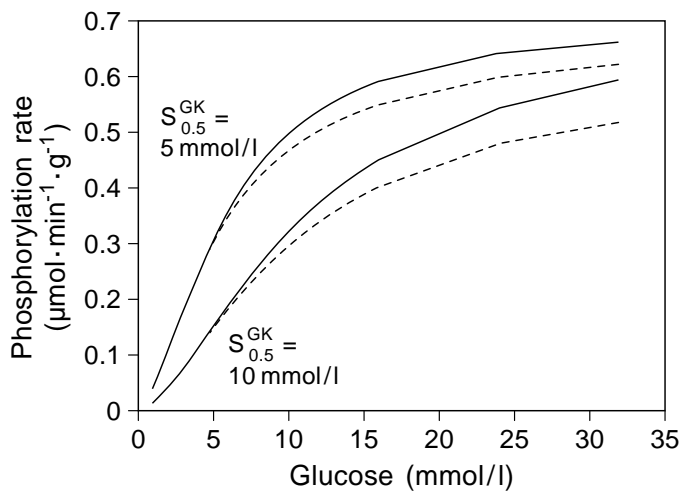

Fig. 6. Effect of the affinity of glucokinase on the glucose phosphorylation rate when G LUT1 (dashed lines) or GLUT2 (solid lines) is used where $S_{0.5}=5 \mathrm{mmol} / \mathrm{l}$ for the top 2 lines and $=10 \mathrm{mmol} / \mathrm{l}$ for bottom 2 lines. The ratio of transport maximal activity (at equilibrium exchange) to that of glucokinase $(G K)$ is 40 . In the phyisologic range $(5-10 \mathrm{mmol} / \mathrm{l})$, the results are nearly identical for the two transporters whereas the phosphorylation rate is very sensitive to the change in affinity of glucokinase

and GLUT2 (Fig.6). Below $10 \mathrm{mmol} / \mathrm{l}$ glucose, at a ratio of $\mathrm{V}_{\text {max }}$ for equilibrium exchange to $\mathrm{V}_{\max }$ of glucokinase of 40 , there is no difference between the phosphorylation rates for the two transporters. In contrast, changing the $S_{0.5}$ of glucokinase from 5 to $10 \mathrm{mmol} / \mathrm{l}$ results in a rightward shift in the doseresponse curve. At higher glucose concentrations, GLUT2 becomes more efficient, suggesting that a beta cell that uses GLUT1 may be more susceptible to impaired glucose sensing at high levels of glucose. Thus, it is the characteristics of glucokinase that determine the dose-response curve.

Implications for non-insulin-dependent diabetes mellitus (NID D M)

A Ithough a number of studies have shown that a loss of GLUT2 is correlated with NIDDM, the kinetic analysis here does not support the notion that losses of GLUT2 can lead to impaired glucose sensing by the islets unless there is a drastic reduction in capacity or affinity. This study and previous analyses [12, 13] have shown that a more than $90 \%$ decrease in capacity and a 10-fold increase in affinity parameter would have to occur to cause a significant decrease in insulin secretion. Therefore, given the low control strength of this step in the transduction pathway of the glucose signal, it is more likely that other steps in glycolysis and the post-metabolic events play a role in the pathogenesis of NIDDM. In part it is the insensitivity of glucose metabolism to glucose transport that could allow for the substitution of a transporter with a much different affinity and properties, G LUT 1, without changing the glucose sensing characteristics of the beta cell.
These conclusions are based on a kinetic analysis of the established mechanisms by which transport and phosphorylation are carried out. Nonetheless, there are some findings that have not been explained on a mechanistic level that we did not address. For instance, there may be a link between G LU T2 expression and the expression of glucokinase [3]; also, the factors that regulate protein levels of GLUT1 and GLUT2 are not understood. If it turns out that GLUT2 has a unique interaction with glucokinase, or G LUT 1 is not maintained at high capacity due to differences in the regulation of its synthesis and degradation, then G LUT2 may indeed be a prerequisite for proper functioning of glucose sensing. However, the results of De Vos et al. [2], if correct, indicate that this is not the case, which is supported by kinetic analysis.

\section{Conclusions}

The traditional view of the role of G LU T 2 in glucose sensing by the pancreatic beta cell is that it must serve to nearly equilibrate the intra- and extra-cellular glucose concentrations so that glucokinase can act to convert the glucose concentration into a phosphorylation signal. From a purely kinetic point of view however, the results of this simulation analysis indicate that GLUT2 is not a necessary component of the glucose sensing apparatus and that G L U T 1 could perform the same function. The salient kinetic properties of GLUT1, its high affinity relative to that of glucokinase and its asymmetry that does not favour transport into the cell, are actually characteristics that make its ability to equilibrate glucose less efficient. However, a sufficiently high capacity of GLUT1 relative to glucokinase will override this. N ote that since both G LUT 1 and G LUT 2 would operate bidirectionally in the beta cell, the maximal activities obtained from zero trans experiments cannot be directly compared to the rate of glucokinase, since the physiologic situation is more closely related to equilibrium exchange conditions. If there is an absolute requirement for GLUT2, it may be in the way protein levels are regulated at the level of gene expression and protein degradation and its possible regulation of glucokinase. It is important to realize that the reason that the requirements for transport kinetics are not so stringent, is that transport does not have a high control strength on rates of glycolysis. Thus, it is the low affinity of glucokinase that allows phosphorylation to be sensitive to changes in glucose in the physiologic range, not the low affinity of transport.

On a parting note, it must not be overlooked that virtually all of the theories explaining glucose sensing by the pancreatic beta cell come from rat and mouse studies, with a few confirmatory experiments in man. 
It would be very interesting, for instance, to know whether these theories would apply to birds, lizards and snakes, whose fasting glucose levels are above $10 \mathrm{mmol} / \mathrm{l}$ [16]. If certain aspects of glucose sensing are conserved across species, this may indicate which steps are essential for function. O n the other hand, nature may have derived more than one mechanism for sensing.

A cknowledgements. These studies were supported in part by National Institute of Health (NIDDK) grants 19525, 22122 and 07314.

\section{References}

1. Matschinsky FM, Ellerman JE (1968) M etabolism of glucose in the islets of Langerhans. J Biol Chem 243: 27302736

2. De Vos A, H eimberg H, Q uartier E, H uypens P, B ouwens L, Pipeleers D, Schuit F (1995) Human and rat beta cells differ in glucose transporter but not in glucokinase gene expression. J Clin Inv 96: 2489-2495

3. Newgard CB (1994) Cellular engineering and gene therapy strategies for insulin replacement in diabetes. D iabetes 43: 341-350

4. Ferber S, R io H B, Johnson J H, et al. (1994) GL U T-2 gene transfer into insulinoma cells confers both low and high affinity glucose-stimulated insulin release. J Biol Chem 269: 11523-11529

5. H eimberg H, D e Vos A, Vandercammen A, Van Schaftingen $E$, Pipeleers D, Schuit F (1993) H eterogeneity in glucose sensitivity among pancreatic $\beta$-cells is correlated to differences in glucose phosphorylation rather than glucose transport. E M B O J 12: 2873-2879
6. Stein WD (1986) Transport and diffusion across cell membranes. A cademic Press, NY

7. Cahill G F J r, A shmore J, E arle A S, Z ottu S (1958) G lucose penetration into liver. A m J Physiol 192: 491-496

8. Carruthers A (1990) Facilitated diffusion of glucose. Phys R ev 70: 1135-1176

9. Williams TF, Exton JH, Park CR, R egen DM (1968) Stereospecific transport of glucose in the perfused rat liver. A m J Physiol 215: 1200-1209

10. Johnson J H, O gawa $A$, Chen L, O rci L, Newgard CB, A lam T, U nger R H (1990) U nderexpression of $\beta$-cell high $\mathrm{K}_{\mathrm{m}}$ glucose transporters in noninsulin-dependent diabetes. Science 250: 546-549

11. G oresky G A, Nadeau BE (1974) U ptake of materials by the intact liver: the exchange of glucose across the cell membranes. J Clin Inv 53: 634-646

12. Sweet IR, M atschinsky FM (1995) M athematical model of $\beta$-cell glucose metabolism and insulin release. I.G lucokinase as glucosensor hypothesis. A m J Physiol 268: E 775E 788

13. Sweet IR, Najafi H, Li G, B erner DK, M atschinsky FM (1996) E ffect of a glucokinase inhibitor on energy production and insulin release in pancreatic islets. A m J Physiol 271: E 606-E 625

14. Lachaal M, Spangler RA, Jung CY (1993) High K m of GLUT 2 glucose transporter does not explain its role in insulin secretion. A m J Physiol 265: E 914-E 919

15. Tal M, Liang $Y$, Najafi H, Lodish HF, Matschinsky FM (1992) Expression and function of GLUT-1 and GLUT-2 glucose transporter isoforms in cells of cultured rat pancreatic islets.J Biol Chem 267: 17241-17247

16. Epple A, B rinn JE (1987) The comparative physiology of the pancreatic islets. Springer, Berlin Heidelberg $\mathrm{New}$ York, p 120 